\title{
Evaluation of Local Recurrence in Giant-Cell Tumor of Bone Treated by Neoadjuvant Denosumab
}

\author{
Pramod Shekarappa Chinder, MBBS, Suraj Hindiskere, MBBS, \\ Srinath Doddarangappa, MBBS, Utkarsh Pal, MBBS \\ Department of Musculoskeletal Oncology, HCG Hospital, Bangalore, India
}

\begin{abstract}
Background: Giant-cell tumor of bone (GCTB) is a locally aggressive primary benign tumor presenting as an expansile osteolytic lesion affecting the epiphysis of long bones. Denosumab halts the osteolysis by giant cells thereby downstaging the tumor, helping in performing less morbid procedures to remove the tumor. Our aim was to report the incidence of local recurrence (LR) in patients operated following neoadjuvant denosumab, to investigate factors associated with LR following extended curettage for GCTB, and to compare the postoperative functional and oncological outcome of patients operated with and without neoadjuvant denosumab. Methods: A total of 123 patients with a mean age of 29.6 years undergoing extended curettage for GCTB were retrospectively divided into group 1 receiving neoadjuvant denosumab and group 2 operated without denosumab. The mean follow-up period was 35 months. The perioperative characteristics and outcome were compared between the two groups and the factors for LR of GCTB were analyzed.

Results: The incidence of LR among patients operated after neoadjuvant denosumab therapy was $42.8 \%$ and was significantly high compared to that in patients without denosumab $(p<0.001)$. On multivariate logistic regression analysis, use of denosumab as a neoadjuvant was the only factor independently associated with $L R$ following surgery $(p=0.002)$. Patients treated with denosumab had a lower LR-free survival rate (log-rank, $p=0.018$ ).

Conclusions: Denosumab was independently associated with increased LR following surgery for GCTB. Denosumab has to be used cautiously in patients in whom the burden of downstaging the disease outweighs the possible chance of LR.

Keywords: Denosumab, Local recurrence, Giant cell tumor, Outcome, Neo adjuvant
\end{abstract}

Giant-cell tumor of bone (GCTB) is a locally aggressive primary benign tumor, presenting as an expansile osteolytic lesion, mainly affecting the epiphysis of long bones. ${ }^{1)}$ GCTB is more prevalent in third and fourth decades of life, commonly occurring around the knee joint and constituting about $20 \%$ of all primary bone tumors. ${ }^{2,3)}$ Surgery is the primary treatment modality for GCTB; salvageable bones are curetted along with use of adjuvants and exten-

Received July 24, 2018; Revised November 4, 2018; Accepted February 27, 2019

Correspondence to: Suraj Hindiskere, MBBS

Department of Musculoskeletal Oncology, HCG Hospital, No. 8, P. Kalinga

Rao Rd, Sampangiram Nagar, Bangalore, Karnataka 560054, India

Tel: +91-98805-53398, Fax: +91-80-2248-5962

E-mail: suraj.ortho@gmail.com sive lesions are resected en bloc. ${ }^{4)}$ The incidence of tumor recurrence following surgery varies between $15 \%$ to $45 \%{ }^{5}$ )

Histologically the tumor tissue is made up of diffusely spread multinucleated giant cells and the neoplastic mononucleated fusiform-shaped stromal cells. ${ }^{6}$ The multinucleated giant cells and their precursors express receptor activator of nuclear factor kappa B (RANK), the activation of which leads to osteoclastic activity and subsequent osteolysis. Denosumab is a fully human monoclonal antibody (IgG2) against RANK ligand with high affinity and specificity, which inhibits the maturation, activation, and survival of the osteoclastic giant cells. ${ }^{7)}$ A benchmark phase II clinical trial showed improvement in quality of life and reduced need for otherwise morbid surgery in patients with GCTB, which resulted in the U.S. Food and Drug Administration's approval of denosumab for GCTB 
Chinder et al. Local Recurrence in Giant-Cell Tumor

Clinics in Orthopedic Surgery • Vol. 11, No. 3, $2019 \bullet$ www.ecios.org

in June 2013. ${ }^{8,9)}$

Denosumab halts the osteolysis by the giant cells at the cellular level and reflects the changes clinically and radiologically, hence it has been used to contain the tumor in order to perform a less morbid procedure. ${ }^{10,11)}$ Though the efficacy of denosumab in downstaging GCTB has been proven beyond doubt, many questions with respect to dosing schedule, duration of treatment, long-term safety profile, and rate of recurrence following tumor removal still remain unanswered. ${ }^{12)}$ The aim of this study was (1) to report the incidence of local recurrence (LR) in patients operated following neoadjuvant denosumab, (2) to investigate factors associated with LR following extended curettage for GCTB, and (3) to compare the postoperative functional and oncological outcome of patients operated with and without neoadjuvant denosumab.

\section{METHODS}

\section{Study Design, Subjects, and Setting}

Prospectively collected database of a tertiary cancer care hospital was retrospectively reviewed, and 219 patients with histopathological diagnosis of GCTB and age more than 16 years, operated between September 2013 and October 2017 were considered for the study. Patients operated following neoadjuvant denosumab therapy were categorized as group $1(n=93)$ and patients operated without denosumab as group $2(\mathrm{n}=126)$. The indications for neoadjuvant denosumab were one or more of the following: (1) Campanacci grade 3 lesions, ${ }^{13)}$ (2) lesions with close proximity to neurovascular bundle, (3) involvement of three or more cortices on orthogonal plain radiographs, (4) open or fungating wound, and (5) significant soft tissue extension. All patients receiving denosumab were counselled regarding the advantages, clinical implications, cost, and possible complications of the therapy. The Institutional Review Board of HCG Hospital approved this study (No. $\mathrm{EC} / 330 / 17 / 03)$. Since this was a retrospective study, consent for participation in the study was not obtained from the patients.

The following patients from both groups were excluded from the study: (1) patients undergoing surgeries other than extended curettage, (2) recurrent GCTB on presentation, (3) prior history of surgery, (4) denosumab used as an adjuvant after surgery, (5) history of radiotherapy, bisphosphonates or any other form of medical management for GCTB, (6) insufficient data, and (7) follow-up less than 1 year after surgery. The remaining 42 patients in group 1 and 81 patients in group 2 were considered for the analysis (Fig. 1).

\section{Description of Treatment}

All included patients either underwent needle biopsy of the presenting osteolytic lesion or had the slides and blocks of biopsy performed elsewhere reviewed at our center, for histological confirmation and evidence of the diagnosis. The demographic details, presenting complaints and duration, Musculoskeletal Tumor Society (MSTS) score, ${ }^{14)}$ blood investigations, radiological investigations (plain radiograph, positron emission tomography, magnetic resonance imaging [MRI] scan, and computed tomography [CT] scan) of the lesion and CT scan of the chest for radiological evidence of pulmonary metastasis were collected for all patients.

For patients in group 1, $120 \mathrm{mg}$ of denosumab was administered subcutaneously after confirmation of normal serum calcium levels. Denosumab was administered once every 4 weeks, with booster doses on day 7 and day 15 of the first month, for a mean duration of 12.3 weeks (range, 1 to 27 weeks), and surgery was performed after a

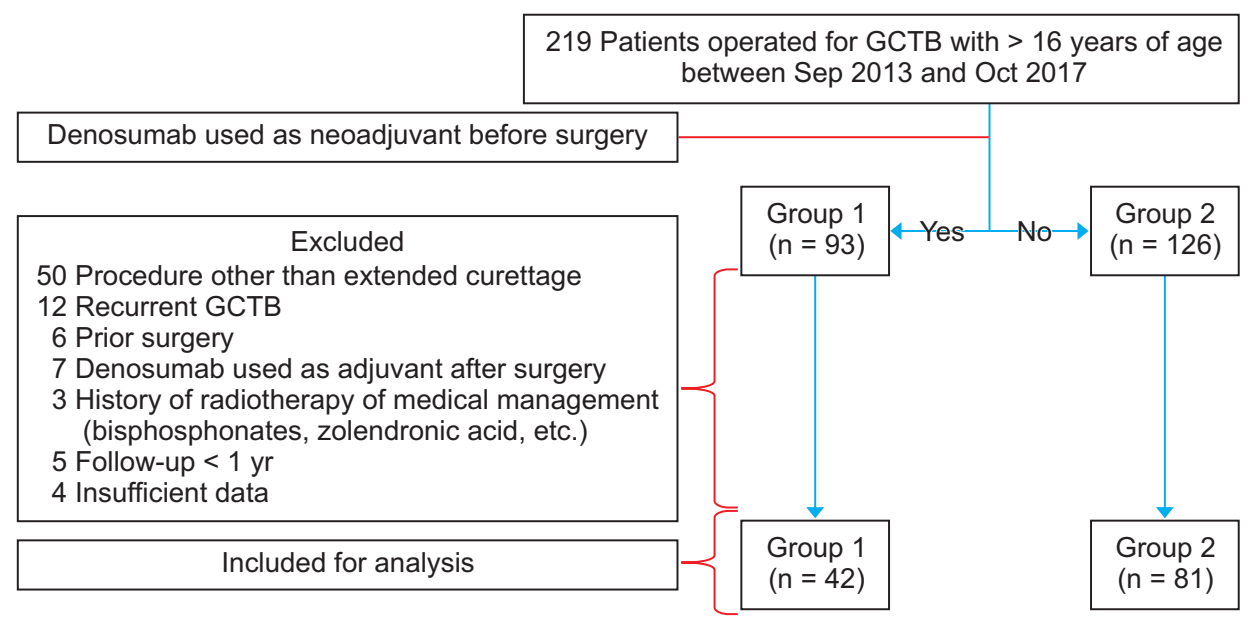

Fig. 1. Flowchart for selection of patients and data collection. GCTB: giant-cell tumor of bone. 
Chinder et al. Local Recurrence in Giant-Cell Tumor

Clinics in Orthopedic Surgery • Vol. 11, No. 3, 2019• www.ecios.org

mean duration of 3.2 weeks following the last dose (range, 2 to 6 weeks). Irrespective of the number of doses of denosumab administered, significant improvement in pain, and reduced size of swelling, regression of osteolysis and evidence of sclerosis in the lesion on radiological examination were considered as end points for surgery.

The surgical procedure (extended curettage) was performed by a single orthopaedic surgeon (PSC) and his

Table 1. Characteristics of Patients with (Group 1) and without (Group 2) Neoadjuvant Denosumab

\begin{tabular}{|c|c|c|c|c|}
\hline Characteristic & All patients $(n=123)$ & Group $1(n=42)$ & Group 2 ( $n=81$ ) & $p$-value \\
\hline Age (yr) & $29.6 \pm 9.8$ & $27.4 \pm 11.6$ & $30.6 \pm 10.4$ & 0.456 \\
\hline Sex & & & & 0.961 \\
\hline Male & $87(70.7)$ & $29(69.0)$ & $58(71.6)$ & \\
\hline Female & $36(29.3)$ & $13(31.0)$ & $23(28.4)$ & \\
\hline Duration of symptom (mo) & $4.6 \pm 3.1$ & $4.8 \pm 3.8$ & $4.3 \pm 2.8$ & 0.425 \\
\hline History of trauma & $28(22.7)$ & $9(21.4)$ & $19(23.4)$ & 0.564 \\
\hline Site & & & & 0.063 \\
\hline Distal femur & $30(24.4)$ & $11(26.2)$ & $19(23.4)$ & \\
\hline Proximal tibia & $24(19.5)$ & $10(23.8)$ & $14(17.2)$ & \\
\hline Distal radius & $14(11.3)$ & $4(9.5)$ & $10(12.3)$ & \\
\hline Pelvis & $12(9.7)$ & $6(14.2)$ & $6(7.4)$ & \\
\hline Foot & $10(8.1)$ & $4(9.5)$ & $6(7.4)$ & \\
\hline Distal tibia & $8(6.5)$ & $1(2.3)$ & $7(8.6)$ & \\
\hline Hand & $8(6.5)$ & $3(7.1)$ & $5(6.1)$ & \\
\hline Distal humerus & $5(4.0)$ & $1(2.3)$ & $4(4.9)$ & \\
\hline Proximal humerus & $5(4.0)$ & 0 & $5(6.1)$ & \\
\hline Proximal femur & $3(2.4)$ & $1(2.3)$ & $2(2.4)$ & \\
\hline Proximal fibula & $2(1.6)$ & 0 & $2(2.4)$ & \\
\hline Distal ulna & $2(1.6)$ & $1(2.3)$ & $1(1.2)$ & \\
\hline Size of lesion $\left(\mathrm{cm}^{3}\right)$ & $3.7 \pm 2.4$ & $4.1 \pm 2.6$ & $3.5 \pm 3.7$ & 0.087 \\
\hline Pathological fracture & $11(8.9)$ & $4(9.5)$ & $7(8.6)$ & 0.233 \\
\hline Pulmonary metastasis & $8(6.5)$ & $3(7.1)$ & $5(6.1)$ & 0.365 \\
\hline Campanacci grade (plain radiograph) & & & & $0.036^{*}$ \\
\hline Grade 1 & $9(5.2)$ & 0 & $9(8.6)$ & \\
\hline Grade 2 & $81(52.0)$ & $25(40.6)$ & $56(59.6)$ & \\
\hline Grade 3 & $33(42.8)$ & $17(59.4)$ & $16(31.7)$ & \\
\hline Serum alkaline phosphatase (IU/L) & $87.3 \pm 35.4$ & $98.3 \pm 21.5$ & $82.3 \pm 30.4$ & 0.096 \\
\hline Serum calcium (mg/dL) & $9.1 \pm 1.7$ & $8.7 \pm 2.1$ & $9.3 \pm 1.7$ & 0.894 \\
\hline Duration of surgery (min) & $103 \pm 42$ & $124 \pm 36$ & $89 \pm 65$ & 0.149 \\
\hline Intraoperative blood loss (mL) & $142 \pm 61$ & $123 \pm 59$ & $156 \pm 47$ & 0.067 \\
\hline Postoperative complication & $7(5.6)$ & $2(4.7)$ & $5(6.1)$ & 0.119 \\
\hline
\end{tabular}


Chinder et al. Local Recurrence in Giant-Cell Tumor

Clinics in Orthopedic Surgery • Vol. 11, No. 3, $2019 \bullet$ www.ecios.org

\section{Table 1. Continued}

\begin{tabular}{|c|c|c|c|c|}
\hline Characteristic & All patients ( $n=123$ ) & Group $1(n=42)$ & Group 2 ( $n=81$ ) & $p$-value \\
\hline \multicolumn{5}{|l|}{ Follow-up } \\
\hline Local recurrence & $33(26.8)$ & $18(42.8)$ & $15(18.5)$ & $<0.001^{*}$ \\
\hline MSTS score at 3 months & $16.4 \pm 3.1$ & $17.0 \pm 3.6$ & $16.1 \pm 3.9$ & 0.621 \\
\hline MSTS score at 6 months & $24.2 \pm 3.1$ & $25.1 \pm 2.8$ & $23.8 \pm 3.6$ & 0.224 \\
\hline MSTS score at 1 year & $28.5 \pm 1.5$ & $29.0 \pm 1.2$ & $28.1 \pm 2.1$ & 0.724 \\
\hline Duration of follow-up (mo) & $35 \pm 20$ & $32 \pm 22$ & $37 \pm 19$ & 0.533 \\
\hline
\end{tabular}

Values are presented as mean \pm standard deviation or number (\%).

MSTS: Musculoskeletal Tumor Society.

*Indicates statistical significance.

team for all the included patients. During the procedure, all visible tumor tissue was thoroughly curetted through a large cortical window, larger than the maximum dimension of the tumor. A high-speed burr was used to confirm no shielded pockets of tumor was left behind. Phenol crystals were used as an adjuvant, which was neutralized with ethanol and finally the walls of the cavity were ablated with an electrocautery. Regular saline and hydrogen peroxide wash was given. Morcellized allograft was impacted to the subchondral region to protect the articular surface and the cavity was either filled with strut allograft supplemented with morcellized allograft or bone cement. Prophylactic surgical stabilization by internal fixation was performed in lesions with risk of developing pathological fracture.

The possible clinicopathological factors associated with development of LR following extended curettage like age, gender, site and size of lesion, symptom and duration on presentation, presence of pulmonary metastasis or pathological fracture on presentation, use of denosumab as a neoadjuvant, preoperative alkaline phosphatase and MSTS score, type of surgery performed and duration of surgery, blood loss, material used to fill the void, and occurrence of postoperative complications were investigated.

\section{Follow-up and Outcome Measures}

During follow-up with the surgical team, plain radiography was performed once in 3 months for the first 2 years followed by once in 6 months till 5 years; MRI scan was performed once in 6 months for the first 2 years followed by once a year till 5 years. If the patient presented with complaints suggestive of LR like new-onset pain or swelling or difficulty in moving the joint anytime during the postoperative period, MRI scan was performed. LR of the disease was defined as evidence of newly appeared lesion on MRI scan reported by a musculoskeletal radiologist, which was absent on prior images following surgery, with or without histopathological conformation in the form of biopsy from the lesion. The duration of first appearance of such lesions following the surgery was noted. The functional outcome in the form of MSTS scores and the oncological outcome in the form of evaluation of LR were analyzed and compared between the two groups.

\section{Statistical Analysis}

Demographic and clinicopathological data were compared between the two groups. Continuous variables were presented as mean with standard deviation and were compared using the independent samples $t$-test. Categorical variables were presented as frequencies with percentage using the Pearson's chi-square test or Fisher exact test. Significance was defined as $p<0.05$. Factors found to have a statistically significant association with the development of LR were included in a multivariate logistic regression analysis. LR-free survival curves were constructed using the Kaplan-Meier method and the differences between survival curves were evaluated by the log- rank test. Statistical analyses were performed using IBM SPSS ver. 23.0 (IBM Corp., Armonk, NY, USA).

\section{Demographics}

Mean age of the patients included in the study was 29.6 years (range, 17 to 49 years). Eighty-seven patients (70.7\%) were male. Of all patients, $22.7 \%(n=28)$ had history of trauma to the site of involvement prior to the onset of symptoms (pain and/or swelling). Distal femur (24.4\%) and proximal tibia (19.5\%) were the most common sites to be involved. About $8.9 \%(\mathrm{n}=11)$ of the patients had pathological fracture and $6.5 \%(n=8)$ of the patients had radiological evidence of pulmonary metastasis on presentation. Mean duration of surgery was $103 \pm 42$ minutes 
Chinder et al. Local Recurrence in Giant-Cell Tumor

Clinics in Orthopedic Surgery • Vol. 11, No. 3, 2019• www.ecios.org

Table 2. Multivariate Logistic Regression Analyses of Factors Associated with Local Recurrence of Giant Cell Tumor of Bone Following Surgery

\begin{tabular}{|c|c|c|c|c|c|c|}
\hline \multirow{2}{*}{ Characteristic } & \multicolumn{3}{|c|}{ Univariate analysis } & \multicolumn{3}{|c|}{ Multivariate analysis } \\
\hline & $\mathrm{OR}$ & $95 \% \mathrm{Cl}$ & $p$-value & $\mathrm{OR}$ & $95 \% \mathrm{Cl}$ & $p$-value \\
\hline Age (yr) & 1.05 & $0.98-1.13$ & 0.177 & & & \\
\hline Sex & & & 0.862 & & & \\
\hline Male & 1 & & & & & \\
\hline Female & 0.87 & $0.34-2.11$ & & & & \\
\hline Duration of symptom (mo) & 0.99 & $0.92-1.06$ & 0.849 & & & \\
\hline History of trauma & & & 0.227 & & & \\
\hline No & 1 & & & & & \\
\hline Yes & 1.87 & $0.67-5.53$ & & & & \\
\hline Site & & & $0.003^{*}$ & & & 0.113 \\
\hline Distal femur and proximal tibia & 1 & & & 1 & & \\
\hline Hand and foot & 3.31 & $2.16-16.74$ & & 2.96 & $1.13-10.36$ & \\
\hline Others & 0.76 & $0.19-8.47$ & & 0.69 & $0.13-6.67$ & \\
\hline Size of lesion $\left(\mathrm{cm}^{3}\right)$ & 1.55 & $0.97-2.47$ & 0.061 & & & \\
\hline Campanacci grade (plain radiograph) & & & 0.138 & & & \\
\hline Grade I & 1 & & & & & \\
\hline Grade II & 2.68 & $0.36-4.36$ & & & & \\
\hline Grade III & 6.31 & $1.42-11.97$ & & & & \\
\hline Denosumab used as neoadjuvant & & & $<0.001^{*}$ & & & $0.002^{*}$ \\
\hline No & 1 & & & 1 & & \\
\hline Yes & 3.89 & $1.16-10.14$ & & 3.12 & $2.48-8.32$ & \\
\hline Pathological fracture & & & 0.124 & & & \\
\hline No & 1 & & & & & \\
\hline Yes & 2.38 & $0.63-6.21$ & & & & \\
\hline Pulmonary metastasis & & & 0.279 & & & \\
\hline No & 1 & & & & & \\
\hline Yes & 1.76 & $0.79-3.33$ & & & & \\
\hline Duration of surgery (min) & 1 & $0.99-1.00$ & 0.432 & & & \\
\hline Intraoperative blood loss (mL) & 0.99 & $0.95-1.04$ & 0.568 & & & \\
\hline Use of cement for reconstruction & & & 0.796 & & & \\
\hline No & 1 & & & & & \\
\hline Yes & 1.34 & $0.32-4.68$ & & & & \\
\hline Postoperative complication & & & 0.133 & & & \\
\hline Yes & 1 & & & & & \\
\hline No & 1.99 & $0.81-4.88$ & & & & \\
\hline
\end{tabular}

OR: odds ratio, $\mathrm{Cl}$ : confidence interval.

*Indicates statistical significance. 
and mean intraoperative blood loss was $142 \pm 61 \mathrm{~mL}$. Following surgery, the patients were followed up for a mean duration of 35 months (range, 8 to 55 months) (Table 1).

\section{RESULTS}

\section{Tumor Response to Denosumab}

In patients who received neoadjuvant denosumab $(\mathrm{n}=$ 42), the median number of denosumab injections administered was 5 (range, 1 to 11) and radiological objective tumor response was seen in $80.9 \%$ of the patients $(n=34)$ as per the inverse Choi criteria. ${ }^{15)}$ Histopathological response to denosumab was seen in $90.5 \%$ of the tissue samples ( $\mathrm{n}$ $=38$ ) obtained during surgery. No patients had histological or radiological evidence of malignant or sarcomatous transformation with denosumab. During the course of the therapy, two patients had hypocalcaemic tetany following administration of denosumab and were managed with intravenous calcium supplementation and supportive care, and three patients had asymptomatic hypocalcaemia and were treated by oral calcium and vitamin $\mathrm{D}$ supplementation.

\section{LR Following Surgery}

The incidence of LR following surgery was $42.8 \%$ ( $\mathrm{n}=$ 18 ) in patients treated with neoadjuvant denosumab and $18.5 \%(\mathrm{n}=15)$ in patients who did not receive denosumab $(p<0.001)$. The mean time to LR following surgery was similar in the two groups $(12.9 \pm 6.5$ vs. $14.3 \pm 4.9$ months, $p=0.343$ ).

In group 1, out of 18 patients with LR, six proximal tibiae, four distal femurs, two pelvises, three feet, two hands, and one distal radius were the involved bones. In group 2, recurrence was evidenced in 15 patients (three distal femurs, three proximal tibiae, two hands, two feet, two distal radii, one pelvis, one distal tibia, and one distal humerus). In view of high LR in small bones of the hand and foot (71.4\% in group 1 and $36.4 \%$ in group 2 ) and knee region (47.6\% in group 1 and $18.2 \%$ in group 2) based on the site of GCTB, all operated patients were divided into group A (distal femur and proximal tibia), group B (hand and foot), and group C (other bones) for analysis of risk factors for LR.

Out of 33 patients with LR, repeat extended curettage was performed in 21 patients and none of these patients received denosumab before the second surgery, and the remaining 12 patients underwent excision of the tumor and four of these patients were administered denosumab before resection to downgrade the tumor. At mean follow-up of $16 \pm 6.3$ months in these 33 patients operated for recurrence, two patients were lost to follow-up, second recurrence was evidenced in $14.2 \%$ of patients undergoing repeat extended curettage $(n=3)$ and none of the patients with excision of the tumor had second recurrence.

\section{Risk Factors for LR of GCTB}

On univariate analysis of possible risk factors for LR following surgery, site of lesion and use of denosumab as a neoadjuvant before surgery were found to be significant $(p<0.05)$. On multivariate logistic regression analysis of these factors, use of neoadjuvant denosumab was found to be the only independent risk factor for $\operatorname{LR}(p=0.002)$ (Table 2).

\section{Outcome}

Average MSTS scores on serial follow-up at 3 months (17.0 [56.7\%] vs. 16.1 [53.7\%]); 6 months, 25.1 [83.7\%] vs. 23.8 [79.3\%]) and at 1 year after surgery $(29.0[96.7 \%]$ vs. 28.1 [93.7\%]) were similar between group 1 and group $2(p>$ 0.05). On Kaplan-Meier analysis, patients with neoadjuvant denosumab showed significantly worse LR-free survival than those operated without denosumab $(p=0.018)$ (Fig. 2).

\section{DISCUSSION}

Denosumab is a fully human monoclonal antibody against RANK ligand preventing osteolytic activity of giant cells, used to contain and downstage GCTB, helping in performing lesser morbid procedures to remove the tumor. Denosumab has been extensively studied and appears effective in optimization of surgery for GCTB, but many questions

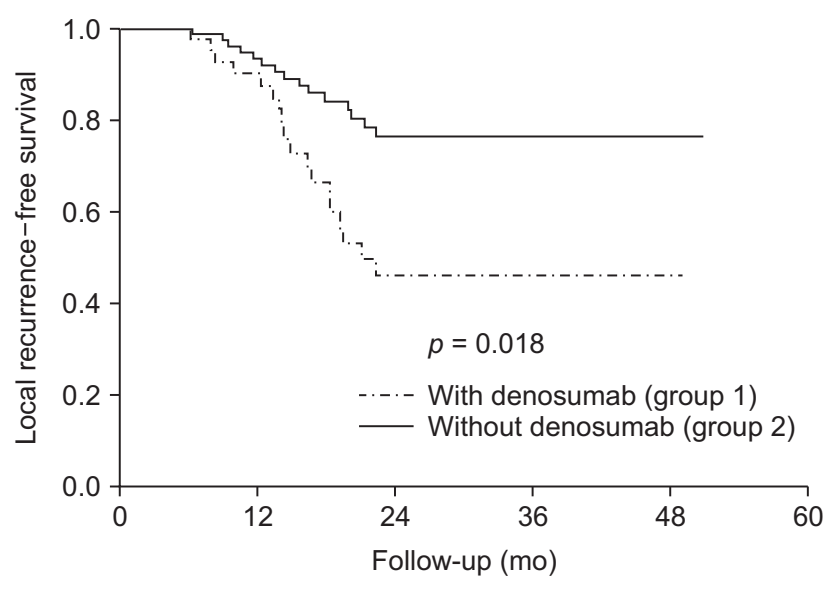

Fig. 2. Kaplan-Meier survival curve for comparing local recurrencefree survival in patients operated for giant-cell tumor of bone, with and without denosumab. 
still remain unanswered regarding the practicality of its usage and there is no data from a large prospective study regarding the recurrence of the tumor in patients receiving denosumab as a neoadjuvant prior to the surgery. ${ }^{4)}$ We report an incidence of 42.8\% (18 / 42) for LR in patients operated by extended curettage for GCTB, with denosumab used as a neoadjuvant. The functional outcome in these patients was similar when compared to patients operated without denosumab but LR-free survival was significantly poor. Use of denosumab as a neoadjuvant before surgery was found to be an independent risk factor for recurrence of GCTB after surgery.

The recurrence of GCTB is highly varied between $12 \%$ and $65 \%{ }^{16)}$ It depends upon multiple factors like the type of surgery performed, adjuvants used, use of cement and location of the tumor. ${ }^{16-21)}$ In an open label phase 2 study by Chawla et al., ${ }^{8)}$ only 22 out of 282 patients enrolled for evaluation of safety and efficacy of denosumab in GCTB underwent surgery. The rate of recurrence following surgery could not be assessed as they were followed up for a mean duration of 9 months. Traub et al. ${ }^{22}$ reported $15 \%$ recurrence in GCTB in their series of 20 patients operated following denosumab for 6 to 11 months and followed up for a median duration of 30 months after surgery. Errani et al. ${ }^{23)}$ reported recurrence in $60 \%(n=15)$ among 25 patients treated by denosumab and curettage for GCTB. Their higher incidence can be attributed to the fact that no adjuvants were used in patients operated by curettage.

On univariate analysis, GCTB of the hand and foot had higher recurrence. Biscaglia et al. ${ }^{24)}$ reported recurrence rate of $30 \%$ following surgery for GCTB of hands and foot. High rates of recurrence of up to $52.6 \%$ has been reported in GCTB of small bones of the hand and foot, as it is postulated that, due to anatomical restraints and in order to maintain function, adequate margins are not obtained during intralesional procedures with gross or microscopic tumor tissue left behind. ${ }^{25-27)}$ Due to the same reason, resection of tumor has lower recurrence when compared to intralesional procedures like curettage, as the chances of microscopic or gross local dissemination of tumor tissue is less likely. ${ }^{27)} \mathrm{Hu}$ et al. ${ }^{17)}$ reported that curettage of GCTB has a hazard ratio of 12.07 (95\% CI, 4.99 to 29.18 ; $p<0.001$ ) over wide excision for developing LR after surgery.

Type of surgery performed has been one of the most important factors determining LR in GCTB. ${ }^{1,2,16,28)}$ Since only patients undergoing extended curettage, using the same adjuvants, operated by a single surgeon were included in our study, nonsurgical factors were better evalu- ated for LR. On multivariate analysis, use of denosumab as a neoadjuvant prior to surgery was the only independent risk factor for LR following surgery. A recent in vitro study demonstrated that on administration of denosumab, the proliferative capacity of the stromal cells is reduced by about $50 \%$ and these neoplastic cells proliferate again once denosumab is discontinued and the microenvironment is favorable. ${ }^{29)}$ However, the RANK ligand expression is completely eliminated, thereby halting osteolysis, and a rim of cortical bone around the tumor and bone septae within the tumor cavity is formed. This hinders the complete removal of the tumor cells that may remain "hidden" within these newly formed bone pockets, thereby increasing LR. The tumor cavity has to be burred multiple times to confirm no tumor pockets are left behind, which can lead to prolonged surgical time and increased intraoperative blood loss if a tourniquet is not used. Errani et al. ${ }^{23)}$ also reported that denosumab was the only independent factor associated with poor recurrence-free survival in patients operated for GCTB, even though many authors felt that the formation of peripheral rim around the tumor helped in easier tumor removal. ${ }^{12,30,31)}$

The patients in our study were matched for the site of lesion and the type of procedure performed, and similar functional outcomes were reported between the two groups at serial follow-up. However, for oncological outcome as evidenced by the Kaplan-Meier curves, the LRfree survival was significantly poor in patients treated with denosumab before surgery (log-rank, $p=0.018$ ). In one of the very few studies evaluating the functional outcome of patients operated following use of denosumab, Deveci et al. ${ }^{32)}$ reported a MSTS score of $87 \%$ in 10 patients with a mean follow-up of 17 months. Denosumab has shown to drastically reduce the clinical symptoms, improve functional outcome, and has also been suggested to be used as a supplement to surgical treatment. ${ }^{33,34)}$

There are some limitations to this study. Firstly, this was a retrospective analysis without randomization of subjects. Denosumab is an expensive drug and only patients consenting for the therapy were administered denosumab and these patients were recruited in group 1. Hence socioeconomic factors were not considered for analysis. Secondly, the patients in group 1 received different number of denosumab injections (range, 1 to 11 ) and this could have been a factor determining the rate of recurrence. Thirdly, patients were followed up for a mean duration of $35 \pm 20$ months. Though it was short, the authors felt it was sufficient as the mean time of recurrence of GCTB after surgery, both in patients receiving and not receiving denosumab is less than 24 months. ${ }^{18,20,23)}$ Lastly, the lesions 
in group 1 could be more aggressive (59.4\% Campanacci grade III) when compared to group 2 (31.7\% Campanacci grade III) and hence the higher recurrence. The authors felt that this is not a confounding factor, as extensive lesions with high chance of LR with extended curettage were treated by excision of the tumor and such patients were excluded from the study.

Neoadjuvant denosumab is associated with increased recurrence following surgery for GCTB and it is the only factor independently associated with lower LRfree survival. Denosumab has to be used cautiously, only when the burden of downstaging the disease to perform a lesser morbid procedure outweighs the possible chance of LR. Further research in the form of prospective clinical trials is needed to establish protocols for the use of denosumab and to evaluate accurate response of GCTB to denosumab.

\section{CONFLICT OF INTEREST}

No potential conflict of interest relevant to this article was reported.

\section{REFERENCES}

1. Mendenhall WM, Zlotecki RA, Scarborough MT, Gibbs CP, Mendenhall NP. Giant cell tumor of bone. Am J Clin Oncol. 2006;29(1):96-9.

2. Rigollino AV, Fernando TS, Tanaka MH, Souza MM. Giant cell tumor locally advanced around the knee: treatment and literature review. Rev Bras Ortop. 2017;52(4):473-8.

3. Lin $\mathrm{F}, \mathrm{Hu} \mathrm{Y}, \mathrm{Zhao} \mathrm{L}$, et al. The epidemiological and clinical features of primary giant cell tumor around the knee: a report from the multicenter retrospective study in china. J Bone Oncol. 2016;5(1):38-42.

4. van der Heijden L, Dijkstra PD, Blay JY, Gelderblom H. Giant cell tumour of bone in the denosumab era. Eur J Cancer. 2017;77:75-83.

5. Chakarun CJ, Forrester DM, Gottsegen CJ, Patel DB, White EA, Matcuk GR Jr. Giant cell tumor of bone: review, mimics, and new developments in treatment. Radiographics. 2013;33(1):197-211.

6. Cowan RW, Singh G. Giant cell tumor of bone: a basic science perspective. Bone. 2013;52(1):238-46.

7. Xu SF, Adams B, Yu XC, Xu M. Denosumab and giant cell tumour of bone-a review and future management considerations. Curr Oncol. 2013;20(5):e442-7.

8. Chawla S, Henshaw R, Seeger L, et al. Safety and efficacy of denosumab for adults and skeletally mature adolescents with giant cell tumour of bone: interim analysis of an open-label, parallel-group, phase 2 study. Lancet Oncol. 2013;14(9):9018.

9. Goldenberg MM. Pharmaceutical approval update. P T. 2013;38(9):518-24.

10. Ueda T, Morioka H, Nishida Y, et al. Objective tumor response to denosumab in patients with giant cell tumor of bone: a multicenter phase II trial. Ann Oncol. 2015;26(10):2149-54.
11. Hakozaki M, Tajino T, Yamada H, et al. Radiological and pathological characteristics of giant cell tumor of bone treated with denosumab. Diagn Pathol. 2014;9:111.

12. Gaston CL, Grimer RJ, Parry M, et al. Current status and unanswered questions on the use of denosumab in giant cell tumor of bone. Clin Sarcoma Res. 2016;6(1):15.

13. Campanacci M, Baldini N, Boriani S, Sudanese A. Giant-cell tumor of bone. J Bone Joint Surg Am. 1987;69(1):106-14.

14. Enneking WF, Dunham W, Gebhardt MC, Malawar M, Pritchard DJ. A system for the functional evaluation of reconstructive procedures after surgical treatment of tumors of the musculoskeletal system. Clin Orthop Relat Res. 1993;(286):241-6.

15. Choi H, Charnsangavej C, Faria SC, et al. Correlation of computed tomography and positron emission tomography in patients with metastatic gastrointestinal stromal tumor treated at a single institution with imatinib mesylate: proposal of new computed tomography response criteria. J Clin Oncol. 2007;25(13):1753-9.

16. Balke M, Schremper L, Gebert C, et al. Giant cell tumor of bone: treatment and outcome of 214 cases. J Cancer Res Clin Oncol. 2008;134(9):969-78.

17. Hu P, Zhao L, Zhang $\mathrm{H}$, et al. Recurrence rates and risk factors for primary giant cell tumors around the knee: a multicentre retrospective study in China. Sci Rep. 2016;6:36332.

18. Gaston CL, Bhumbra R, Watanuki M, et al. Does the addition of cement improve the rate of local recurrence after curettage of giant cell tumours in bone? J Bone Joint Surg Br. 2011;93(12):1665-9.

19. Kivioja $\mathrm{AH}$, Blomqvist $\mathrm{C}$, Hietaniemi $\mathrm{K}$, et al. Cement is recommended in intralesional surgery of giant cell tumors: a Scandinavian Sarcoma Group study of 294 patients followed for a median time of 5 years. Acta Orthop. 2008;79(1):86-93.

20. Prosser GH, Baloch KG, Tillman RM, Carter SR, Grimer RJ. 
Does curettage without adjuvant therapy provide low recurrence rates in giant-cell tumors of bone? Clin Orthop Relat Res. 2005;(435):211-8.

21. Leggon RE, Zlotecki R, Reith J, Scarborough MT. Giant cell tumor of the pelvis and sacrum: 17 cases and analysis of the literature. Clin Orthop Relat Res. 2004;(423):196-207.

22. Traub F, Singh J, Dickson BC, et al. Efficacy of denosumab in joint preservation for patients with giant cell tumour of the bone. Eur J Cancer. 2016;59:1-12.

23. Errani C, Tsukamoto S, Leone G, et al. Denosumab may increase the risk of local recurrence in patients with giant-cell tumor of bone treated with curettage. J Bone Joint Surg Am. 2018;100(6):496-504.

24. Biscaglia R, Bacchini P, Bertoni F. Giant cell tumor of the bones of the hand and foot. Cancer. 2000;88(9):2022-32.

25. Rajani R, Schaefer L, Scarborough MT, Gibbs CP. Giant cell tumors of the foot and ankle bones: high recurrence rates after surgical treatment. J Foot Ankle Surg. 2015;54(6):11415.

26. Shigematsu K, Kobata Y, Yajima H, Kawamura K, Maegawa N, Takakura Y. Giant-cell tumors of the carpus. J Hand Surg Am. 2006;31(7):1214-9.

27. Co HL, Wang EH. Giant cell tumor of the small bones of the foot. J Orthop Surg (Hong Kong). 2018;26(3):2309499018801168.

28. Cheng DD, Hu T, Zhang HZ, Huang J, Yang QC. Factors affecting the recurrence of giant cell tumor of bone after surgery: a clinicopathological study of 80 cases from a single center. Cell Physiol Biochem. 2015;36(5):1961-70.

29. Mak IW, Evaniew N, Popovic S, Tozer R, Ghert M. A translational study of the neoplastic cells of giant cell tumor of bone following neoadjuvant denosumab. J Bone Joint Surg Am. 2014;96(15):e127.

30. Muller DA, Beltrami G, Scoccianti G, Campanacci DA, Franchi A, Capanna R. Risks and benefits of combining denosumab and surgery in giant cell tumor of bone-a case series. World J Surg Oncol. 2016;14(1):281.

31. Goldschlager T, Dea N, Boyd M, et al. Giant cell tumors of the spine: has denosumab changed the treatment paradigm? J Neurosurg Spine. 2015;22(5):526-33.

32. Deveci MA, Paydas S, Gonlusen G, Ozkan C, Bicer OS, Tekin M. Clinical and pathological results of denosumab treatment for giant cell tumors of bone: prospective study of 14 cases. Acta Orthop Traumatol Turc. 2017;51(1):1-6.

33. Martin-Broto J, Cleeland CS, Glare PA, et al. Effects of denosumab on pain and analgesic use in giant cell tumor of bone: interim results from a phase II study. Acta Oncol. 2014;53(9):1173-9.

34. Sorensen AL, Hansen RL, Jorgensen PH. Denosumab may be a supplement to the surgical treatment of giant cell tumours of bone. Ugeskr Laeger. 2016;178(36). pii: V03160204. 